\title{
Claude Vincenot: PRÉCIS DE GRAMMAIRE LOGIQUE. Honoré Champion, Paris, 1998
}

»Précis de grammaire logique« des Autors Claude Vincenot ist ein monumentales Werk auf 1209 Seiten. Es enthält mehrere Teile, diese zerfallen in mehrere Kapitel. Der erste Teil beschäftigt sich mit binären Oppositionen und mit dem Identitätsprinzip, der zweite Teil mit verschiedenen Dimensionen der syntagmatischen Achse, der dritte Teil mit lexikalischen Analysen, der vierte Teil mit logisch-grammatischen Analysen und der fünfte und zugleich letzte Teil hat zum Gegenstand die Verbvalenz. Es folgen noch Bibliographie, Index und Inhaltsverzeichnis.

Diese Grammatik ist besonders interessant auch für einen slowenischen Germanisten wegen der vielen slowenischen Belege und weil die Art und Weise, wie sie sich mit verschiedenen grammatischen und linguistischen Problemen im Allgemeinen auseinandersetzt, Parallelen zu Methoden in einigen germanistischen linguistischen Monographien (vgl. z.B. Harald Weinrich, Textgrammatik der deutschen Sprache, Mannheim 1993) ziehen lässt. Ich werde deshalb versuchen, das Werk auch unter diesem besonderen Gesichtspunkt zu besprechen.

Der erste Teil handelt u.a. von den phonologischen Applikationen (35 ff.) und von Oppositionen (55) und bringt dazu auch slowenische Belege $(37,48)$. Bei der Analyse der morphosyntaktischen Applikation von Personalpronomina gibt es sogar einen Versuch, anthropologisch vorzugehen (1.1.3.1.A.a.). Es imponiert die permamente Unterscheidung von syntaktischen, semantischen und stilistischen Ebenen des Sprachsystems in ihrem Zusammenspiel $(108,119)$.

Das Unterkapitel 2.1.2.2.B (120 ff.) (»endophore syntaxique) ist lesenswert, weil es ein Problem thematisiert, das auch im Deutschen vorkommt: die Asemantizität des unpersönlichen Pronomens il (es). Auch das Problem des sog. Objektsprädikativs (auf Französisch attribut du sujet-objet - 131) ist in beiden Sprachen ähnlich. Der verbale Charakter des Attributs (132) ist richtig dargestellt, es gibt da Parallelen zum inneren Objekt (133), das sich schon an der Grenze zur Stilistik bewegt. Auch die Frage der syntaktischen und semantischen Kongruenz $(174,149)$ sowie des Tempusgebrauchs in auf die Vergangenheit bezogenen Relativsätzen (157) zeigt sich in französischer wie auch in deutscher Sprache ähnlich schwierig.

Gegenstand des 2. Teiles sind syntagmatische und paradigmatische Relationen. Der 2. Teil berührt auch das Problem der Wortbildungskategorie Wortbildungsnest (Fleischer, Wolfgang/Barz, Irmhild, Wortbildung der deutschen Gegenwartssprache, Tübingen 1992) (lourd, lourdeur, lourdement, alourdir), im Grunde genommen einer besonderen Art der Wortfamilie. Die Rede ist auch von dem leeren Subjekt il (mit Parallele zum Slowenischen, 217). Das 5. Kapitel dieses 2. Teiles spricht von der Zweideutigkeit von Genitivattributen (vgl. 269, 564, 1007). Bei Nachverben, die auch im Deutschen vorkommen, gibt es wiederum eine Parallele zum Slowenischen (297 ff.). Erstaunlich viele Parallelen zum Slowenischen gibt es auch in Kapiteln XI (L'accord, 
phénomène de dépendance linéaire), XII (L'accord du participe passé avec un pronom antéposé) und XIII (L'analyse dépendancielle), und zwar auf Seiten 386 ff., 394, 397, 402, 408, 427 f., 430 f., 451, 454.

Der dritte Teil bringt lexikalische Analysen. Der Autor geht an die Klärung der Begriffe wie Wortfeld, semantisches Feld, lexikalisches Feld, Semasiologie, Onomasiologie, Wortbildung und der damit verbundenen Probleme strukturalistisch heran.

Im Kapitel zur Synonymie behandelt er auch die Stilfiguren wie Metapher, Metonymie, Synekdoche, und zwar mit und ohne monosemantische Reduktion.

Einmalig ist die Erklärung des Mechanismus, nach welchem die Bezeichnungsübertragung der Metapher (convergence metaphorique) funktioniert. Die einzelnen Phasen dieser Übertragung sind klar präsentiert und voneinader abgegrenzt und auch entsprechend belegt. Ein Beweis, dass man auch stilistischen Fragen mit strukturalistschen Methoden nachgehen kann. (Wiederum ein slowenischer Beleg dazu auf S. 591.)

Im dritten Teil sind überzeugend die Analysen verschiedener lexikalischer Felder (649). Im Rahmen der Wortbildungsproblematik ist nicht zu übersehen, dass der Autor auch sog. Blockierungen anführt und sich mit dem Problem umfassend beschäftigt. (Ein slowenisch-russicher Beleg dazu auf S. 673.)

Der vierte Teil bringt lexisch-grammatische Analysen der nominalen und akjektivischen Funktionen sowie der Adverbialsätze. Einige Probleme aus diesem Teil: Subjekt des Imperativs (695), Inversion (704), eine Art Determinantionsgefüge (nach Weinrich 1993) (la relation du support et de l'apport) (775), Motivation (813 u. passim), Temporalsätze (auch ein slowenischer Beleg auf S. 829), thematische und rhematische Kausalität (842 ff.) u.a.m.

In diesem Zusammenhang drängt sich die kritische Überlegung auf, ob man das französiche Wort peut-être als Adverb klassifizieren kann (auf Deutsch Modalwort, slowenisch naklonska beseda).

Der 5. Teil widmet sich unter verschiedenen Gesichtspunkten der Verbvalenz.

Die Fakten, die diese »logische« Grammatik auszeichnen, sind die folgenden:

Es ist eine umfangreiche Grammatik, die fast alle Probleme der Grammatik behandelt. Alle Ebenen des Sprachsystems - von der Phonetik und Phonologie bis hin zu textlinguistischen Aspekten (die Rede ist z. B. auch von den Begriffen wie Anapher (139), Katapher (718), Endophora $(102,119,126)$, Exophora $(152,238)$, Deixis (104, 121, 139), Referenz (102, 1137), Metareferenz (418), Antezedens (103), Präsuppositionen (654)) - sind erfasst, darüber hinaus auch Stilistik und Pragmatik.

Es ist zwar eine auf Französisch verfasste Grammatik, sie bringt aber Belege aus vielen anderen europäischen und nichteuropäischen Sprachen (der slowenischen, russischen, deutschen, englischen, lateinischen usw.), so dass es sich im Grunde genommen um eine allgemeine Grammatik mit konfrontativen und kontrastiven Zügen handelt.

Es ist eine verständlich geschriebene Grammatik, die auch komplizierte Probleme anschaulich (mit vielen Tabellen, Graphen, Diagrammen usw.) verständlich deutet. 
Es ist eine moderne Grammatik, die alles auch in strukturalistischer Optik darzustellen vermag, womit sie die Anwendung der Sprache in computerunterstützten Situationen ermöglicht.

Stojan Bračič

\section{Nina Janich: WERBESPRACHE. EIN ARBEITSBUCH. Gunter Narr Verlag, Tübingen, 1999}

Gegenstand des zu besprechenden Buches von Nina Janich ist das Phänomen Werbesprache, „das nicht nur als Kulisse ... überall präsent ist ..., sondern das auch immer mehr Kult- und Kunststatus und damit ausdrückliche Aufmerksamkeit erhält" (7). Wer sich heute mit der Sprache beruflich beschäftigt und sie analysiert, kommt um die Werbesprache nicht herum: fast alle modernen Tendenzen der Sprachentwicklung lassen sich daran nachweisen, und wenn Werbesprache aus inflationären Grunden nicht gerade als Hauptkorpus für Analysen ausgesucht wird, so kann man kaum der Versuchung widerstehen, für gewisse Hypothesen mindestens sporadisch doch auch werbesprachliches Belegmaterial heranzuziehen. Bislang hat es kein Werk gegeben, das sich so systematisch und umfassend mit der Werbesprache beschäftigt wie das Arbeitsbuch von Nina Janich. Es bringt einleitend einen theoretischen Rahmen über „Markt und Kommunikation“, präsentiert danach die Bausteine der Werbung, untersucht Werbetexte unter verschiedenen sprachwissenschaftlichen Forschungsaspekten, um mit methodischen Tipps zu Analysen und einigen Überlegungen zur Diachronie und zu den Fragen der Interkulturalität in Werbeuntersuchungen zu schließen. Das Buch von Nina Janich ist, wie bereits oben ausgeführt, als ein Arbeitsbuch konzipiert, das bedeutet, das zu jedem Kapitel Fragen formuliert sind, die zu vertiefender Auseinandersetzung mit der Materie anregen sollen. Lösungsvorschläge zu den Aufgaben befinden sich im Anhang.

In dem einleitenden, mehr theoretischen Kapitel (11 ff.) werden die Geschichte der Sprachwissenschaft rund um die Werbesprache und deren Desiderate diskutiert. Im Anschluss daran wird Werbung definiert (16) und einige grundlegende Elemente dieses Phänomens (Werbeobjekte, Werbeziele, Werbewirkung, Zielgruppenbestimmung, Werbemittel, Werbeträger) beleuchtet. Vor dem Hintergrund verschiedener Kommunikatiosmodelle wird Werbesprache als „eine inszenierte Form von Kommunikation“ präsentiert. Das tiefgreifende Dilemma zwischen Manipulation und/oder Information wird aufgeworfen.

Im sog. „Mikrokosmos Anzeige“ (40) werden die wesentlichen Bausteine der Werbung dargestellt: Schlagzeile, Fließtext, Slogan, Logo, Produktname, besondere Formen 